\title{
The role of steroids in the development of post-partum mental disorders
}

\author{
Andrea Paskovaa , Roman Jirakc, Michaela Mikesovaa , Karolina Adamcovaa , Zdenka Fartakovaa , Vladimira Horakovaa, \\ Michal Kouckya, Martin Hilla, ${ }^{\mathrm{a}}$, Hana Hruskovicova ${ }^{\mathrm{b}}$, Luboslav Starka ${ }^{\mathrm{b}}$, Michaela Duskovab ${ }^{\mathrm{b}}$, Antonin Parizek ${ }^{\mathrm{a}}$
}

\begin{abstract}
Background. Unfavorable post-partum changes to mental well-being affect more than half of all women, and are a risk to the health of both mother and baby. Their effects place strains on health and social systems. Currently, no generally accepted theory exists of the causes and mechanisms of post-partum mental disorders.

Methods. Literature search up to 2012, using PubMed and search words: neuroactive steroids, post-partum mental disorders, depression, corticotropin-releasing hormone and estrogens.

Results. There are several theories for post-partum depression. One is that autoimmune diseases are involved. Others revolve around genes responsible or that lead to increased disposition to the disorder. It is likely however that the process is associated with the separation of the placenta and the fetal zone of fetal adrenal gland, the main sources of corticotropin-releasing hormone and sexual and neuroactive steroids during pregnancy, and the ability of the receptor system to adapt to these changes. The central nervous system is able to produce neurosteroids, but the drop in levels of peripheral steroids likely leads to a sudden deficit in neuroinhibitory steroids modulating ionotropic receptors in the brain.

Conclusions. Post-partum depression is a multifactorial disease with unknown etiology. It is probably associated with sudden changes in the production of hormones influencing the nervous system, and on the other hand the ability of the receptor system to adapt to these changes. When the relative changes in concentrations of hormones, rather than their absolute levels, is likely more important.
\end{abstract}

Key words: neuroactive steroids, postpartum depression, corticotropin-releasing hormone, estrogens, allopregnanolone

Received: April 15, 2012; Accepted: October 18, 2012, Available online: November 12, 2012

http://dx.doi.org/10.5507/bp.2012.098

${ }^{a}$ Department of Obstetrics and Gynecology, $1^{\text {st }}$ Faculty of Medicine and General Teaching Hospital in Prague, Czech Republic bInstitute of Endocrinology, Prague

'Department of Psychiatry, $1^{\text {st }}$ Faculty of Medicine, Charles University in Prague and General University Hospital in Prague Corresponding author: Antonin Parizek, e-mail: parizek@porodnice.cz

\section{INTRODUCTION}

Steroid hormones act through two different pathways. The classic effect is at the genomic level, acting over slow time scales of hours to days. The non-genomic effect of steroids on the other hand can influence cellular processes in seconds or even milliseconds. Steroid hormones that act in this non-genomic way and influence neural excitability are known as neuroactive steroids. Those that are produced in the brain are called neurosteroids. The molecular mechanisms for these direct effects, influencing neural stimulation through the modulating function of various ligands activating ion channels, were already described in the 1980s and 90s. These steroids also act as transcription factors. For this reason their activity has been studied in psychological and neurological disorders, mostly in depressive and anxiety disorders, reactions to stress, premenstrual tension, epilepsy, alcoholism, and in cognitive function disorders including Alzheimer's disease.

\section{NEUROACTIVE STEROIDS AND DEPRESSION}

It has been demonstrated that neurosteroids play a large role in the onset, course, and treatment of depression. In animal models, it has been shown that administering the antidepressant fluoxetine, a member of the class of selective serotonin reuptake inhibitors (SSRI), markedly increases allopregnanolone levels in the brain ${ }^{1}$. In contrast, in animals with induced depressive symptoms directly given allopregnanolone, the symptoms of depression lessened ${ }^{2}$. Severe depression in people is associated with lowered brain levels of allopregnanolone and simultaneously increased levels of tetrahydrodeoxycorticosterone (THDOC), and treatment with fluoxetine increases the levels of allopregnanolone ${ }^{3}$. SSRI-type antidepressants likely increase the levels of allopregnanolone through the activation of enzymes involved in the synthesis of 3-alphareducing neurosteroids ${ }^{4}$.

It is also surmised that the additional neurosteroids: dehydroepiandrosterone (DHEA), dehydroepiandrosterone sulfate (DHEAS), and pregnenalone sulfate (PS) have antidepressant effects in humans and in animal models. Clinical studies have not been unequivocal however, 
and DHEA is not recommended for treating depression or for augmenting antidepressant therapy according to evidence-based medicine. Even the brain concentration levels of DHEA in depressive patients are equivocal.

A very important discovery was that neurosteroids may change the excitability of neuronal interactions with specific neurotransmitter receptors. Most attention has been given to the receptor for gamma-aminobutyric acid receptor (GABA) type $\mathrm{GABA}_{\mathrm{A}}$, an ionotropic receptor associated with a chloride ion channel. After ligand binding, GABA leads to hyperexcitation of the neuronal membrane, preventing further stimulation. GABA $A_{A}$ receptors are inhibitory, but the GABA system is not always inhibitory. In the fetus, it is stimulatory. These receptors are created from various subunits (alpha, beta, gamma, and others). The chloride channel is made of a combination of five subunits. The binding site for GABA is between subunits alpha and beta. The $\mathrm{GABA}_{\mathrm{A}}$ receptor is allosterically influenced by various types of substances. Benzodiazepines interact with the combination of subunits (alpha1)2 (beta2)2 gamma2, and with other combinations ${ }^{5,6}$. In addition to benzodiazepine, the $\mathrm{Z}$ hypnotics (zolpidem, zopislon, zaleplon, etc) also bind to this site. Other allosteric sites, working at the level of the chloride channel, are the barbiturate site, where barbiturates bind, the site for general anesthesia, where for instance propotil, steroids, halotan and ethylalcohol bind, and the so called picrotoxin site, where for instance the agonist clomethiazol binds.

Some neurosteroids act as substances modulating the $\mathrm{GABA}_{\mathrm{A}}$ receptors, binding to these receptors allosterically. Dehydroepiandrosterone (DHEA), which is synthesized in brain astrocytes and elsewhere, has an an-

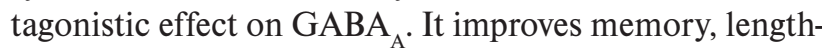
ens REM sleep (during which long-term memories are stored). Decreased levels of DHEA are generally found in depressed patients, and its administration improves the symptoms of depression and cognitive function ${ }^{7,8}$. Similar effects have been found for dehydroepiandrosterone sulfate (DHEAS).

Severe depression in humans is characterized by an imbalance in endogen neurosteroids. The levels of allopregnanolone and some other alpha-reduced nuerosteroids are reduced in the brain fluid as well as the plasma. Allopregnenolone is an allosteric modulator of the $\mathrm{GABA}_{\mathrm{A}}$ receptor. It has been shown that modulation of the $\mathrm{GABA}_{A}$ receptor has great importance for the onset of depression and for its treatment. Some synthetic neurosteroids will likely be used as new antidepressants ${ }^{9}$.

\section{POST-PARTUM MENTAL DISORDERS}

Post-partum mental disorders (PPMD) occur in some form in more than half of women. In most cases these are temporary, without long-term negative consequences. However, there remain a relatively significant proportion of women for whom the complications lead to aftereffects for themselves and even for their children. Some of these negative effects can appear with a significant delay, even in puberty or adulthood. In the most serious cases, mental disorders associated with the end of pregnancy can lead to tragic consequences, including suicide of the mother or infanticide. In any case, post-partum mental disorders are a significant risk to the health of the mother and child, and dealing with these disorders has long been a strain on health and social systems.

PPMD can be divided into three categories: Postpartum blues (PPB) occurs in 50-70\% of mothers and usually disappears by itself within a week without the need for specific therapy. Mothers suffering from PPB experience a temporary phase of emotional weakness around the second to fifth day after birth. This temporary psychopathological PPB is similar to premenstrual tension, with irritability being the main symptom. In fact PPB is not depression, but a sudden and unexpected fluctuation in mood including anxiety, discouragement, perplexity, lack of concentration, and forgetfulness. The etiology of PPB has not yet been elucidated, though many hypotheses have been proposed ${ }^{10}$.

Post-partum depression (PPD), occurring in 10-15\% of births, is a significant depressive state occurring most frequently between the fourth and sixth week of pregnancy ${ }^{11}$. It is estimated that almost half of these cases go undocumented. PPD manifests as symptoms of depressive moods, sleep disorders, anxiety, loss of interest and psychological imbalance, feelings of guilt, even suicidal thoughts ${ }^{12}$. Post-partum psychosis (PPP) (occurrence of about $0.1-0.2 \%$ ) is most often the result of bipolar and schizophrenic disorders. They require hospitalization due to the risk of suicide or infanticide ${ }^{12}$.

\section{CORTICOTROPIN-RELEASING HORMONE AND POST-PARTUM DEPRESSION}

Despite the existence of some hypotheses, there is no current generally accepted theory of the causes and mechanisms of post-partum mental disorders ${ }^{13-15}$. The separation of the placenta and the fetal compartment of the fetal adrenal gland likely plays an important role in the mechanism of PPMD, since they are the main sources of corticotropin-releasing hormone $(\mathrm{CRH})$ and steroids during pregnancy. $\mathrm{CRH}$ has been identified in various organs and systems including the immune system and reproductive systems of both sexes. CRH immunoreactivity has been documented in peripheral sites of inflammation, the ovaries, endometrium, placenta, deciduum, and testes. In addition, $\mathrm{CRH}$ plays a direct immunomodulatory role as an autocrine/paracrine mediator of inflammation, and regulates reproductive functions including inflammatory components, such as ovulation, luteolysis, decidualization, and implantation. Placental CRG thus influences the physiology of pregnancy and the onset of birth ${ }^{16}$.

$\mathrm{CRH}$ is primarily a regulator of the hypothalamo-hypophyseal-adrenal axis (HPA), which is closely associated with stress. The activity of HPA is markedly increased in patients with depression. Various related hypothalamic 
peptidergic systems have close connections to the symptoms of depression. Increased production of vasopressin, which further stimulates the release of ACTH induced $\mathrm{CRH}$ in depression and which has been colocalized in neurons producing $\mathrm{CRH}$ and neuronal nucleus supraopticus (SON), are associated with suicide risk. The increased activity of oxytocin producing neurons in the paraventricular nucleus (PVN) can be related with disorders in food intake. On the other hand, the suprachiasmatic nucleus (SCN), which functions as a biological clock of the brain, shows lowered production of vasopressin in depression, which could explain sleep problems in depressed patients as well as play a role in the marked activation of $\mathrm{CRH}$ (ref. $\left.{ }^{17}\right)$.

\section{STEROIDS AND POST-PARTUM DEPRESSION}

When the placenta is discharged during birth, the source of almost all pregnancy $\mathrm{CRH}$ and placental steroids, i.e. estrogen, progesterone, and its neuroactive reduced metabolites, is also removed. In addition, the source of precursors to placental steroids, the fetal zone of the fetal adrenal gland, is also removed. The most abundant pregnancy $3 \beta$-hydroxy-5-en steroids produced here, i.e. pregnenolone sulfate a dehydroepiandrosterone sulfate, are also neuroactive steroids. Long-term extreme increased levels of neuroinhibitory pregnane steroids in the periphery evidently lead to their more intense penetration across the hematoencephalic barrier to the central nervous system (CNS), where they induce negative compensation mechanisms, consisting of lowered affinity of the $\mathrm{GABA}_{\mathrm{A}}$ receptor for neuroinhibitory pregnane steroids. The $\mathrm{CNS}$ is able to produce a certain amount of its own neuroactive steroids ${ }^{18}$, but despite this, a decrease in the levels of peripheral steroids could produce a sudden deficit of neuroinhibitory steroids acting mainly through the modulation of $\mathrm{GABA}_{\mathrm{A}}-\mathrm{r}$. The question remains how quickly can ionotropic receptors in the brain adapt to the significantly lowered influx of steroid modulators from the periphery, and whether possible defects in this synchronization may be related to manifestation of PPMD, as some studies have indicated ${ }^{19}$. Other studies have found increased prenatal levels of progesterone in women with PPD compared to controls, and a quicker decrease in these levels to levels comparable ${ }^{20}$ or even lower ${ }^{21}$ than controls.

Neuroinhibitory pregnane steroids may also mediate the inhibition of 5-hydroxy-tryptamine neurons through modulation of the $\mathrm{GABA}_{\mathrm{A}}-\mathrm{r}$, which could be significant for the relationships of changes in mood to changes in reproductive hormones, such as in PPD (ref..$^{22}$ ). They are also increased along with the amount of dopamine released in the nucleus accumbens, and thus likely influence mood and motivation ${ }^{23}$.

There currently exists only one study on the relationship between pregnane steroids and PPMD. Allopregnanolone, progesterone, cortisol, prolactin and estradiol were measured in 40 healthy primiparas after the birth of healthy children. On the same day, each volunteer answered the "Hamilton Rating Scale for Depression" questionnaire for psychometric evaluation and the "Stein Questionnaire" for symptoms of PPB (ref. ${ }^{24}$ ). 18 women (45\%) were evaluated as being PPB positive. These women had lowered levels of allopregnanolone, though the corresponding differences in progesterone levels were not statistically significant. The levels of progesterone were correlated in PPB-negative women, though not in PPBpositive. There was a significant negative correlation found between Hamilton score and levels of allopregnanolone and progesterone ${ }^{24}$.

In addition to progesterone and its neuroactive metabolites, estrogen can also be associated with the etiology of PPMD. Estrogens influence the mental state of memory through classical monoamine and neurotransmitter receptor mechanisms in the brain, and low levels of estrogens are associated with PPD. Furthermore, estrogens stimulate an increase in dopamine receptors, and increase the density of 5-hydroxy-tryptamine binding sites in brain regions controlling mood, mental status, cognitive functions, emotions and behavior.

In rodents, estrogens also stimulate the expression of the arginine-vasopressin gene in the stria terminalis, a region storing social and olfactory memories. Described mechanisms indicate the protective role of estrogens ${ }^{25}$, which has been supported by their success in the treatment of women with PPD ( ref. $^{26,27}$ ), as well as the negative effects observed when estrogen applications were interupted in studies of women ${ }^{28}$.

Results from one study show a drop in the activity of brain cortical sulfatase and growth in the concentration of DHEAS in this brain region after suddenly ending estradiol in rats, which could lead to depressed activity in $\mathrm{GABA}_{\mathrm{A}}$-r. The authors speculate on the relevance of this phenomenon to PPMD (ref. ${ }^{29}$ ).

The role of estrogen in the development of post-partum depression has led to considerations of its use in treatments. Ahokas et al. tried giving estrogen substitution to women with PPD who were hypogonadal. After one week, depressive symptoms were reduced by half ${ }^{30}$. Gregoire et al. undertook a placebo-controlled study, in which women with PPD were given transdermal $17 \beta$-estradiol (200 ug daily) and cyclic dydrogestenone (10 mg per day, 12 days per month), or a placebo. After a month of treatment the women taking estrogens had a four-fold reduction in depressive symptoms compared to those taking the pla$\mathrm{cebo}^{31}$. Due to a lack of experience, however, estrogen substitution is considered a complimentary method to conventional treatments with anti-depressives. The results of studies so far indicate that the response to estrogen substitution is rapid, with minimal side-effects, and minimal transfer to mother's milk, giving hope for the role of estrogens in the treatment of PPD (ref. ${ }^{32}$ ). 


\section{CONCLUSIONS}

The etiology of post-partum depression is as yet unknown. It is clear that it is a multifactorial disease, with various internal and external factors playing a role. Despite several hypotheses, there is no generally accepted theory on the causes and mechanisms of post-partum mental disorders. It is probable however, that the process is associated with sudden changes in the production of hormones influencing the nervous system, and on the other hand the ability of the receptor system to adapt to these changes. The relative concentrations of hormones, rather than their absolute levels, is likely most important.

\section{ACKNOWLEDGEMENTS}

This work has been supported by grant IGA MZ CR no NS 9790-4, NT 12211-5 and NT 13890, by the project MZ CR for conceptual development of research organization 00023761 (Institute of Endocrinology, Prague, Czech Republic) and SVV UK 2012 no 264501.

Authorship contributions: APas: literature search and manuscript writing; MM, KA, ZF, VH, MK: literature search of the area obstetrics; $\mathrm{HH}$ MD, MH: literature search of the area steroids and manuscript writing; RJ, LS, APar: final approval.

Conflict of interest statement: The authors stated that there are no conflicts of interest regarding the publication of this article.

\section{REFERENCES}

1. Uzunov DP, Cooper TB, Costa E, Guidotti A. Fluoxetine - elicited changes in brain neuroactice steroid kontent measured by negative ion mass fragmentography.; Proc Natl Acad Sci U S A 1996;93:12599604.

2. Khisti RT, Chopde CT. Serotonergic agents modulace antidepressantlike effect of the neuroactive steroid 3alfa-hydroxy-5alfa-pregnan20-one in mice. Brain Research 2000;868:291-300.

3. Strohle A, Pasini A, Romeo E, Hermann B, Spalletta G, di Michele F, Holsboer F, Rupprecht R. Fluoxetine decreases concentrations of 3alfa,5alfa-tetrahydrodeoxycorticosterone (THDOC) in major depression. Journal of Psychiatric Research 2000;34:183-6.

4. Mellon SH, Griffin LD, Compagnone NA. Biosynthesis and action of neuroactive steroids. Brain Research Reviews 2001;37:3-12.

5. Froestl W. Chemistry and pharmacology of GABAB receptor ligands. Adv Pharmacol 2010;58:19-62.

6. Baur R, Kaur KH and Sigel E. Structure of alfa6beta3delta GABAA receptors and their lack of etanol sensitivity. Journal of Neurochemistry 2009;111:1172-81.

7. Baulieu EE, Thomas G, Legrain S, Lahlou N, Roger M, Debuire B, Faucounau V, Girard L, Hervy MP, Latour F, Leaud MC, Mokrane A Pitti-Ferrandi $\mathrm{H}$, Trivalle $\mathrm{C}$, de Lacharrière $\mathrm{O}$, Nouveau $\mathrm{S}$, RakotoArison B, Souberbielle JC, Raison J, Le Bouc Y, Raynaud A, Girerd X and Forette F. Dehydroepiandrosterone (DHEA), DHEA sulfate, and aging: Contribution of the DHEAge Study to a sociobiomedical issue. PNAS 2000;97:4279-84.

8. Reddy DS. Neurosteroids: Endogenous role in the human brain and the therapeutic potentials. Prog Brain Res 2010;186:113-37.
9. Schüle C, Eser D, Baghai TC, Nothdurfter C, Kessler JS, Rupprecht P. Neuroactive steroids in affective disorders: target for novel antidepressant or anxiolytic drugs? Neuroscience 2011;191:55-77.

10. Lanczik M, Brockington IF. Postpartum dysphoric syndrome. Psychopathology, diagnosis and etiology. Fortschr Neurol Psychiatr 1999;67:60-7.

11. Godfroid IO, Charlot A. Postpartum psychiatry. Rev Med Brux 1996;17:22-3

12. Kemp B, Bongartz K, Rath W. Psychic disturbances in the postpartum period: an increasing problem? Z Geburtshilfe Neonatol 2003;207:159-65.

13. Gleicher N. Postpartum depression, an autoimunne disease? Autoimmun Rev 2007;6:572-6.

14. Mitchell C, Notterman D, Brooks-Gunn J, Hobcraft J, Garfinkel I, Jaeger K, Kotenko I, McLanahan S. Role of mother's genes and environment in postpartum depression. Proc Natl Acad Sci USA 2011;17:8189-93.

15. Maguire J, Mody I. GABAA plasticity during pregnancy: relevance to postpartum depression. Neuron 2008;59:207-13.

16. Kalantaridou S, Makrigiannakis A, Zoumakis E, Chrousos GP. Peripheral corticotropin-releasing hormone is produced in the immune and reproductive systems: actions, potential roles and clinical implications. Front Biosci 2007;12:572-80.

17. Swaab DF, Bao AM, Lucassen PJ. The stress system in the human brain in depression and neurodegeneration. Ageing Res Rev 2005;4:141-94.

18. Stoffel-Wagner B.Neurosteroid biosynthesis in the human brain and its clinical implications. Ann NY Acad Sci 2003;1007:64-78.

19. Beckley $\mathrm{EH}$, Finn DA. Inhibition of progesterone metabolism mimics the effect of progesterone withdrawal on forced swim test immobility. Pharmacol Biochem Nebav 2007;87:412-9.

20. Chang J, Zhang Y, Cui L. Impact of placental hormone withdrawal on postpartum depression. Zhonghua Fu Chan Ke Za Zhi 1995;30:342-4.

21. Harris B, Lovett $L$, Newcombe RG, Read GF, Walker R, Riad-Fahmy D. Maternity blues and major endocrine changes: Cardiff puerperal mood and hormone study II. Bmj 1994;308:949-53.

22. Kaura V, Ingram CD, Gartside SE, Zouny AH, Judge S.J. The progesterone metabolite allopregnanolone potentiates GABA(A) receptor-mediated inhibition of 5-HT neuronal activity. Eur Neuropsychopharmacol 2007;17:108-15.

23. Rouge-Pont F, Mayo W, Marinelli M, Gingras M, Le Moal M, Piazza PV). The neurosteroid allopregnanolone increases dopamine release and dopaminergic response to morphine in the rat nucleus accumbens. Eur J Neurosci 2002;16:169-73.

24. Nappi RE, Petraglia F, Luisi S, Polatti F, Farina C, Genazzani AR. Serum allopregnanolone in women with postpartum "blues". Obstet Gynecol 2001;97:77-80.

25. Fink G, Sumner BE, Rosie R, Grace O, Quinn JP. Estrogen control of central neurotransmission: effect on mood, mental state, and memory. Cell Mol Neurobiol 1996;16:325-44.

26. Ahokas A, Kaukoranta J, Aito M. Effect of oestradiol on postpartum depression. Psychopharmacology (Berl) 1999;146:108-10.

27. Gregoire AJ, Kumar R, Everitt B, Henderson AF, Studd JW. Transdermal estrogen for treatment of severe postnatal depression. Lancet 1996;347:930-3.

28. Bloch M, Schmidt PJ, Danaceau M, Murény J, Nieman L, Rubinow DR. Effects of gonadal steroids in women with a history of postpartum depression. Am J Psychiatry 2000;157:924-30.

29. Maayan R, Strous RD, Abou-Kaoud M, Weizman A. The effect of 17 beta estradiol withdrawal on the level of brain and peripheral neurosteroids in ovarectomized rats. Neurosci Lett 2005;384:156-61.

30. Ahokas A, Kaukoranta J, Wahlbeck K, Aito M. Estrogen deficiency in severe postpartum depression: successful treatment with sublingual physiologic 17beta-estradiol: a preliminary study. J Clin Psychiatry 2001;62(5):332-6.

31. Gregoire AJ, Kumar R, Everitt B, Henderson AF, Studd JW. Transdermal oestrogen for treatment of severe postnatal depression. Lancet 1996;347:930-3.

32. Moses-Kolko EL, Berga SL, Kalro B, Sit DK, Wisner KL. Transderma estradiol for postpartum depression: a promising treatment option. Clin Obstet Gynecol 2009;52:516-29. 